


\section{Surgimiento y Desarrollo de la Educación Superior, en correspondencia al bicentenario de la Independencia de Centroamérica y la reforma de Córdoba, y perspectivas para el 2030}

Emergence and Development of Higher Education, in correspondence with the bicentennial of the Independence of Central America and the reform of Córdoba, and prospects for 2030

\section{RESUMEN}

El presente estudio trata de dar a conocer las causas que dieron origen a la independencia de Nicaragua al momento histórico, así como las consecuencias de la reforma universitaria de córdoba y cómo la universidad del siglo XXI, se enrumba hacia nuevo horizonte de la cualificación del nuevo profesional ciudadano capaz de resolver problemas sensibles a la sociedad, así como también hacer un ejercicio reflexivo hacia lo emergente, hacia los retos y desafíos de la universidad de acuerdo a la agenda 2030. Se ha podido analizar de manera inductiva y analítica la información, considerando que las causas que detonaron el manifiesto de la independencia, no están tan lejos a los actuales, y si bien es cierto que la Colonia se vivió del siglo XV al XVII en plena vigencia física, hoy en día en el siglo XXI, se mantienen algunos rezagos de dominación, de imperio, de arrogancia, de discriminación, de deseos libertad, de opresión, de explotación de esclavitud (Véase concepto de revolución de Fidel Castro pág. No 2). De igual modo las causas del manifiesto de Córdoba son por las mismas causas de independencia de la universidad, primero organizada y dominada por la iglesia bajo sus intereses, luego por el estado de igual manera dirigida por y para las élites. Es por tanto que estos acontecimientos históricos y heroicos de los pueblos cada uno en su momento y su contexto han posibilitado el desarrollo holístico de la universidad y se está cambiando todo lo que hay que cambiar. Que hay problemas aún sin resolver, si hay problemas, pero vamos adelante, hacia mejores estadios de desarrollo, revolucionando continuamente. Entiéndase de calidad. Y Como dice Fidel en su concepto de Revolución: "es luchar con audacia, inteligencia y realismo; es no mentir jamás ni violar principios éticos; es convicción profunda de que no existe fuerza en el mundo capaz de aplastar la fuerza de la verdad y las ideas". Conclusivamente se logran evidenciar que desde la colonia se practican por parte de Europa colonial y otros imperios del norte vejámenes que violenta los derechos humanos: como la discriminación, el desconocimiento de la verdad, la manipulación para sojuzgar, el uso de la mentira como arma de miedo y terror, el elitismo, la prepotencia; sin embargo el lado opuesto de la práctica colonizadora, los aprendizajes, los errores, cometidos y observados han permitido significativos avances en las universidades moderna como en lo académico, lo investigativo, el extensionismo y su impacto social la internacionalización, el desarrollo tecnológico, la autoevalaución y la acreditación nacional e internacional, son logros evidentes de desarrollo revolucionario de las universidades de Nicaragua, en Centroamérica y América Latina y el Caribe.

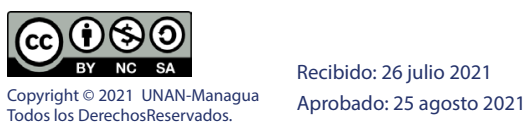

\section{PALABRAS CLAVE}

Reforma de Córdoba, Acta de

Independencia, Desarrollo Universitario,

Calidad, Educación superior 


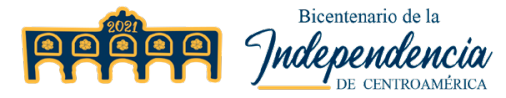

\section{ABSTRACT}

The present study tries to make known the causes that gave rise to the independence of Nicaragua at the historical moment, as well as the consequences of the university reform of Córdoba and how the university of the 21st century, it is routed toward a new horizon of qualification of the new professional citizen capable of solving problems sensitive to society, as well as doing a reflective exercise toward the emerging, the challenges and challenges of the university of according to the 2030 agenda.It has been possible to investigate by triangulating the information, that the causes that detonated the manifesto of independence, not so far from the current ones, and although it is true that the Colony was lived from the 15th to the 17th century in full physical force, today In the 21st century, there are still some lags of domination, empire, arrogance, discrimination, desires for freedom, oppression, exploitation of slavery. In the same way, the causes of the Córdoba manifesto are for the same causes of independence of the university, first organized and dominated by the church under its interests, then by the state in the same way directed by and for the elites. It is therefore that these historical and heroic events of the peoples each in their time and their context have made the holistic development of the university possible and everything that needs to be changed is being changed. That there are still unsolved problems, there are problems, but we are moving forward, towards better stages of development, continually revolutionizing. Understand quality. And As Fidel says in his concept of Revolution: "it is to fight with audacity, intelligence and realism; it is never lying or violating ethical principles; it is a deep conviction that there is no force in the world capable of crushing the force of truth and ideas". In conclusion, it is possible to show that from the colony, colonial Europe and other northern empires practice humiliations that violate human rights: such as discrimination, ignorance of the truth, manipulation to subjugate, the use of lies as a weapon of fear and terror, elitism, arrogance; However, the opposite side of the colonizing practice, the learning, the errors, committed and observed have allowed significant advances in modern universities such as academics, research, extensionism and its social impact internationalization, technological development, self-assessment and national and international accreditation are evident achievements of revolutionary development of the universities of Nicaragua, in Central America and Latin America and the Caribbean.

\section{KEYWORDS}

\author{
Cordoba Reform, Independence Act, \\ University Development, Quality, Higher \\ education
}

\section{Introducción.}

El creciente desarrollo económico social a partir del siglo XVIII en Nicaragua, después de la independencia de Centroamérica, ha dejado herencias culturales, sociales, económicas y políticas importantes que surgen después de la creación de la educación superior, cuya ideología, su filosofía, estructura y su desarrollo orgánico funcional ha posibilitado llegar hasta donde estamos, conociendo su contribución en estos aspectos. Situación que se mejora en el siglo XIX con el manifiesto de córdoba. Hoy en el siglo XXI es el momento para repensar sobre lo emergente, hacia dónde va la Educación Superior del país y cómo está respondiendo a la agenda 2030 y Plan Nacional de Desarrollo Humano. 


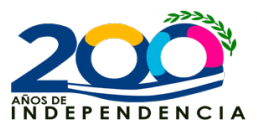

El trabajo aquí expuesto se plantea en tres momentos importantes que han surgido en este Bicentenario de la independencia de Centroamérica el Primero de la Colonia y el origen de las Universidades en 1812 a 1878 , el segundo momento interesante para las universidades latinoamericanas fue la reforma de Córdoba en 1918 y la etapa del siglo XX. Era de la tercera revolución industrial. Además se describe de manera sintética la evolución organizativa funcional de la Universidad en Nicaragua, con algunos esbozos a nivel latinoamericano, lo que por razones de ontogenia son inseparables y su relación con el manifiesto de Córdoba y el manifiesto de la Independencia de Centroamérica/Latinoamérica.

Su fundamentación está explícita en internet, así se hizo una búsqueda completa en varios motores de Google sobre el tema y resulta que existe poca información confiable y detallada sobre el asunto. La información se analizó mediante métodos lógicos y cualitativo, para hacer un análisis más completo de las teorías propuestas y a partir de ellos, establecer aproximaciones importantes al menos sobre el primer periodo, para las dos etapas siguientes si hay una vasta información.

\section{Objetivos.}

1. Caracterizar el desarrollo de las universidades en América Latina y el Caribe y específicamente en Nicaragua, en tres etapas: Colonia (1812), Reforma de Córdoba (1918) y etapas del S XX y XXI

2. Compartir cuáles serán los retos y desafíos de la Educación Superior de cara a la agenda 2030

Reflexionemos. ¿Por qué surge el Manifiesto de Córdoba en Argentina y ¿Por qué surge el manifiesto de la independencia de Centroamérica?, ¿Acaso la sociedad demandó en cada momento de esta autonomía? ¿Ha avanzado la universidad en su estructura y funcionamiento en función del desarrollo social?

\section{Fundamentación teórica.}

Al mencionar revolución traigo acá el concepto de revolución del Comandante Fidel, al respecto dice: ...."El proceso que va progresivamente transformando la sociedad en todos sus aspectos de manera integral y justa, creado las condiciones para garantizar con el esfuerzo de todos o la mayoría la satisfacción de las necesidades materiales y espirituales de cada individuo". Del Prado. N (2016)

Es sentido del momento histórico;
es cambiar todo lo que debe ser cambiado;
es igualdad y libertad plenas;
es ser tratado y tratar a los demás como seres humanos;
es emanciparnos por nosotros mismos y con nuestros propios esfuerzos;
es desafiar poderosas fuerzas dominantes dentro y fuera del ámbito social y nacional;
es defender valores en los que se cree al precio de cualquier sacrificio;
es modestia, desinterés, altruismo, solidaridad y heroísmo;
es luchar con audacia, inteligencia y realismo;
es no mentir jamás ni violar principios éticos;
es convicción profunda de que no existe fuerza en el mundo capaz de aplastar la fuerza de la verdad y las ideas.
Revolución es unidad, es independencia, es luchar por nuestros sueños de justicia para Cuba y para el mundo, que
es la base de nuestro patriotismo, nuestro socialismo y nuestro internacionalismo."


El Comandante Fidel dejó un concepto dialéctico, diáfano y previsor en el momento histórico como el de hoy, sobre revolución.

Las Universidades en el mundo y en particular la Latinoamericana y específicamente la centroamericana hoy en día han alcanzado un alto grado de desarrollo poniendo en práctica esos valores evocados por Fidel en: Desarrollo en estructuras de gobierno, en cobertura estudiantil, en desarrollo tecnológico, en formación del profesorado, en la gestión del posgrado, la investigación-innovación, la extensión, en la internacionalización, es decir cambios y desarrollo en búsqueda de la calidad.

Bernheim en su obra La Universidad ante los retos del siglo XXI señala que la mayoría de los estudiantes (De hijos de adinerados)salía al exterior a estudiar medicina, Entonces quiénes estudiaban medicina en esa época las élites, tal como lo señala El sociólogo alemán Hanns-Albert Steger Citados por Tünnermann B. C 199,p.122-126) sostiene que tal vez este proceso se comprenda mejor, "si consideramos que la conquista se pude entender como una repetición, en la medida en que estaba investida con el carácter de una cruzada, de la Reconquista de la Península Ibérica que acababa de terminar". siempre pensaron en una confederación o reunión de "reinos cerrados en sí mismos, que se mantenían unidos por la Corona, y no por una administración central. De ahí que, posteriormente, esta noción condujo al pronto establecimiento de universidades en los Nuevos Reinos y sustentó las voces que en todos los virreinatos, capitanías generales y audiencias pedían la creación de universidades cuando "aún olía a pólvora y todavía se trataba de limpiar las armas y herrar los caballos" le agregaría "y los indios mutilados, vejados, explotados y esclavizados todavía limpiaban sus latigazos y heridas en su cuerpo y el dolor en su alma", según la frase del cronista Vázquez.

Entre otras características de esta universidad, es que el latín era el idioma universitario, era requisito de ingreso a cualquier Facultad. El método de enseñanza consistía en la lectio o lectura viva voice por el catedrático o lector del texto señalado, seguida de las correspondientes explicaciones.

Situación que aún se mantiene en ciertas universidades en pleno siglo XXI. Fortalecida esta idea con el paradigma Napoleónico en las universidades que ha perdurado y anclado en el ejercicio docente por siglos.

La lectio se complementaba con la disputatio, que activaba la docencia por la participación de los estudiantes en la formulación de objeciones o argumentos en relación con las conclusiones que proponía el profesor. Los actos de conclusiones eran programados periódicamente y representaban una oportunidad para ejercitar las capacidades dialécticas de los estudiantes, principalmente en material como teología y filosofía.

Carlos Tünnermann en su libro: Breve historia del desarrollo de la universidad en América Latina expone que la primera universidad erigida por los españoles en el Nuevo Mundo fue la de Santo Domingo, en la Isla Española (28 de octubre de 1538). La última fue la de León de Nicaragua, creada por decreto de las Cortes de Cádiz el 10 de enero de 1812. Entre ambas fechas sumaron 32 las fundaciones universitarias, si bien algunas, como la de la Plata o Charcas (Bolivia); la de Mérida (Yucatán, México) y la de Buenos Aires (Argentina) sólo existieron de Jure, pues no llegaron a funcionar plenamente antes de la conclusión del periodo colonial. 
Esta universidad su oferta educativa estaba dirigida a satisfacer las demandas de los

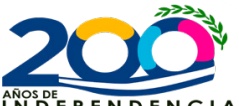
banqueros españoles y peninsulares, además de ofertar las carreras de medicina, teología y derecho, con una matrícula muy pobre proveniente de las élites de la época.

Bernheim en su obra La Universidad ante los retos del siglo XXI. Estructura y forma de gobierno de la Educación Superior en Nicaragua. 1812 a 1878. La Universidad en Nicaragua, nace formalmente en el año 1812, gracias a la gestión del Padre Ayestas y el Dr Tomas Ruiz quienes intercedieron ante la corte de Cádiz quien concedió al Colegio Tridentino en León la categoría de Universidad Plena. Esta es una evidencia de que la Iglesia estaba a cargo de la educación y de la educación superior en el país y en Latinoamérica, esto muestra que el enfoque educativo que se brindaba en esa época era de índole religioso. La estructura de gobierno era muy pobre, rígida y dirigida por un sacerdote autorizado por la Iglesia y dependía fundamentalmente de la aprobación del papa o del Rey.

De igual modo se contaba con una pobre matrícula estudiantil. Desde 1812 al 1888 año en que se separa la universidad de la iglesia y se establecieron en Granada y León, ambas ofrecían sólo dos carreras:

Medicina, Teología y Derecho. Se dice que la sociedad de ese entonces necesitaba de estas dos ciencias:

1. 1) Derecho, porque los banqueros (supuesta demanda social) querían proteger sus activos y enviando a las universidades a sus hijos o allegados resolverían su situación legal y garantizar sus inversiones.

2. 2) Medicina era una carrera de moda en todo el continente latinoamericano y caribeño, y por otro lado la realidad de país, es que no contaban con los médicos que al menos se acercaran a la relación de un médico por cada 1000 habitantes tanta población. 3) La Teología por el hecho de mantener la presencia de novicios encargados de culturizar a los peninsulares y criollos e un acercamiento con el nuevo mundo y España, es decir, mantener y ampliar la hegemonía de la Corona en Iberoamérica y una forma de hacer una reconquista.

\section{Figura $\mathrm{N}^{\circ}$ 1. Organización de la Universidad en periodo colonial.}

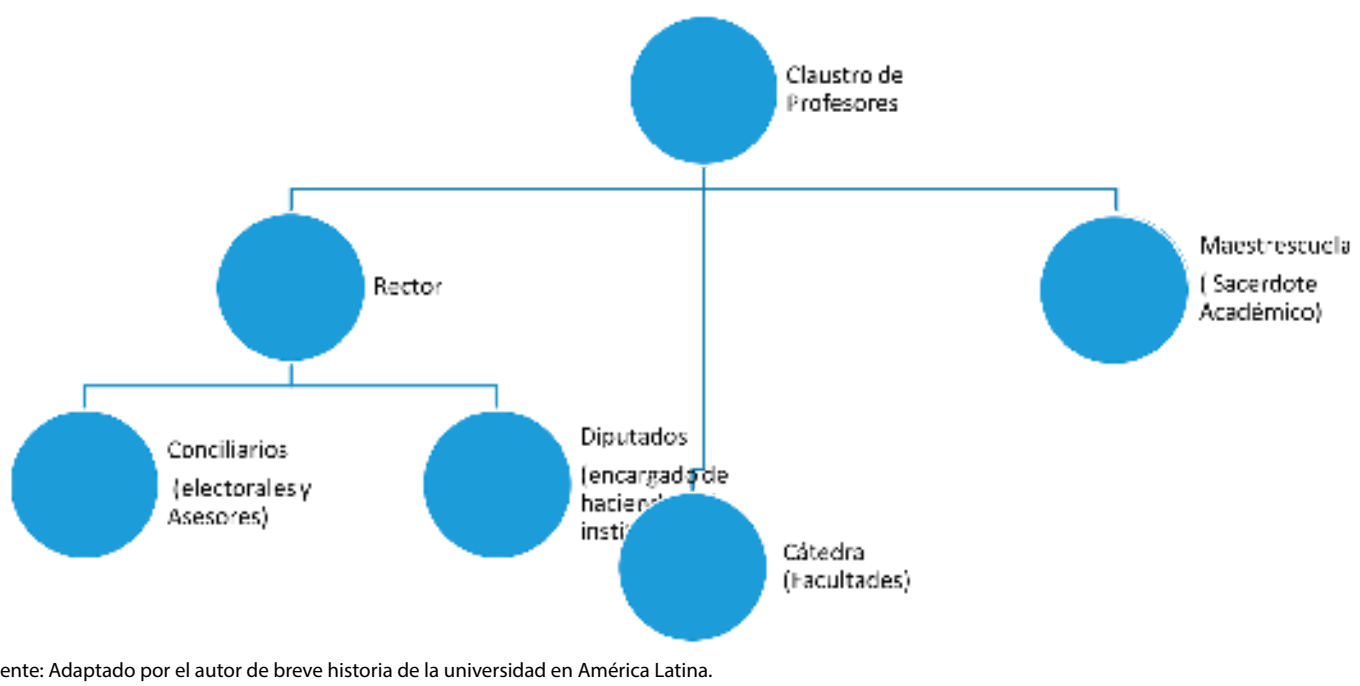


La organización y estructuras académicas de Salamanca, reproducidas luego con muy pocas modificaciones por sus filiales americanas, podemos describirlas, en pocas palabras, de la manera siguiente:

Se puede observar que la máxima autoridad de la universidad estaba gobernada por el claustro de profesores a quien le correspondían la dirección superior de la enseñanza y la potestad para formar los estatutos. El rector estaba asesorado además por los Conciliarios (Clérigos designados por la iglesia) y Diputados, instancias encargadas de orientar, sugerir, propones y recomendar al Rector en materia académica y administrativa-financiera.

\section{Desarrollo de las universidades en Nicaragua y Latinoamérica entre 1878 y 1944.}

Organizativamente la universidad entre los años 1893, tiempos del gobierno liberal de José Santos Zelaya la universidad era dirigida por un rectorado, con un enfoque centralista de la administración educativa, en los años (1893-1909) se realizó una reforma al régimen administrativo de la universidad, siguiendo el modelo francés conocido como Napoleónico. Éste consistió en la división por facultades y escuelas independientes entre sí que se regían bajo currículos educativos propios de las áreas de estudios, pero correspondían a un modelo educativo general que estaba en vigencia y que reflejaba el contexto socio-cultural, político e ideológico.

En los años Durante el gobierno de Santos Zelaya se consolidan los avances universitarios hasta que derrocado Zelaya con la influencia y el beneplácito de los EEUU cierran de nuevo la Universidad en 1913 (Intervención de los marines hasta 1926 y de 1927 a 1933), actos seguidos del destierro e incluso asesinatos de profesores y personas ligadas al mundo de la cultura. Más tarde, por Decreto No. 446 del 27 de marzo de 1947 la Universidad de León se transforma en Universidad Nacional.

El 5 de mayo de 1966 se declara oficialmente por decreto 105 de la Constitución Política de la República de Nicaragua, "Universidad Nacional Autónoma de Nicaragua". Desde su fundación la sede de la UNAN ha sido la ciudad de León con dependencias universitarias en Managua, Carazo y Estelí. En 1982 se decreta la separación de la Universidad Nacional Autónoma de Nicaragua (UNAN) con asiento en León en dos núcleos, uno en León y otro en Managua con rectores independientes. La atención universitaria entre 1950 y 1957 oscilaba entre 490 estudiantes en 1950, 950 estudiantes en el año 1952 y en 1957 bajó nuevamente a 919, en promedio se matricularon 726 estudiantes; sin embargo, la población de jóvenes con edad de estudiar en la universidad andaba por un promedio de 167,572 jóvenes en edad de estudios universitarios

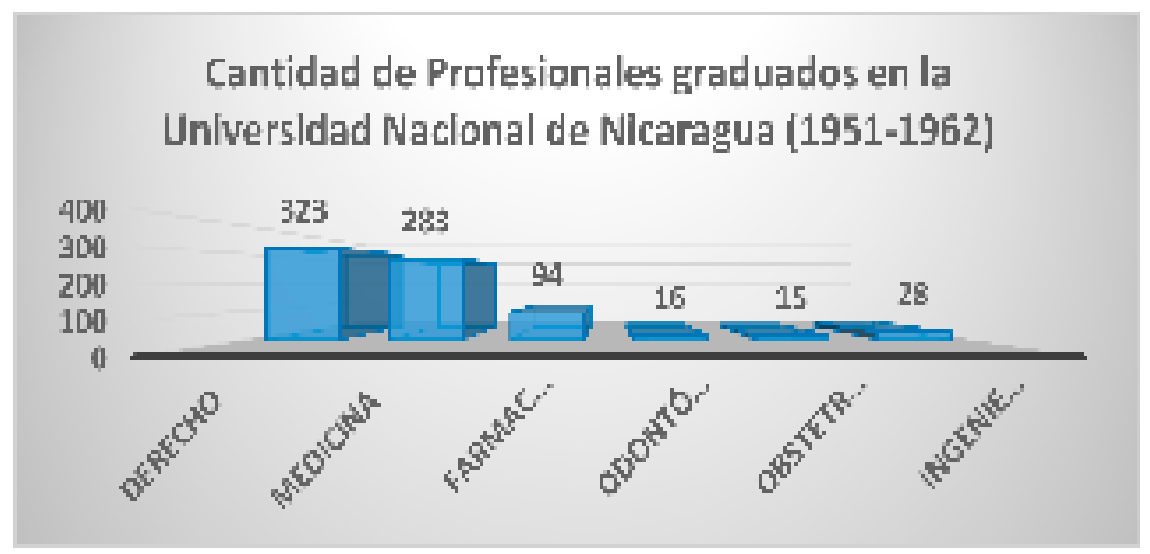

Profesionales graduados entre 1951-1962 


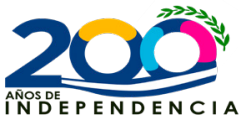

(18 a 25 años de edad) lo que demuestra que la demanda satisfecha era apenas el $0.4 \%$, si los datos los hubiésemos calculado anualmente la cobertura sería de 0.004\%. Ramírez. S (1971)

Continúa exponiendo Ramírez, que los profesionales graduados en la Universidad Nacional de

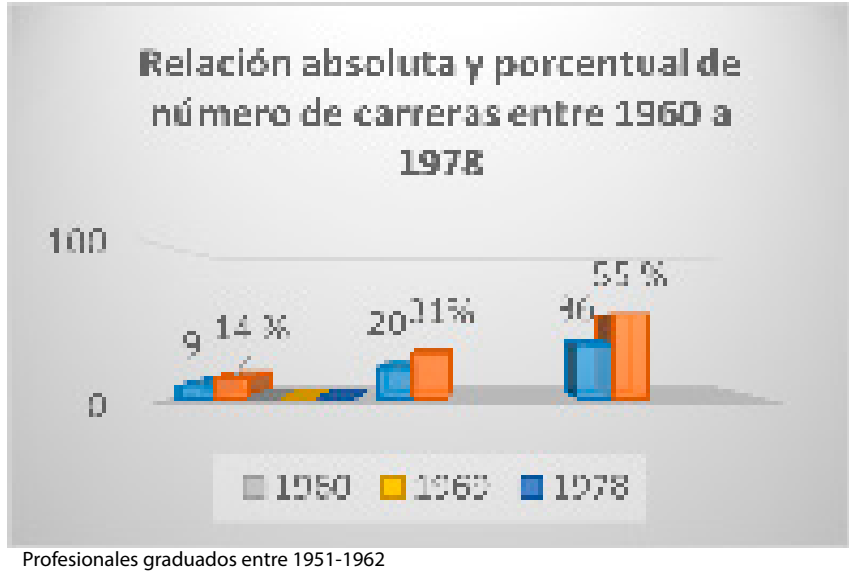

Nicaragua, entre 1951 y 1962 había un total de 759 profesionales; 323 Licenciados en Derecho, 283 en Medicina, 94 Farmacéuticos, 16 odontólogos, 15 médicos obstetras y 28 apneas ingenieros civiles. CNE (1996)

Dado el interés del país de fortalecer la producción del algodón y el café que el boom en ese periodo, los empresarios demandaban técnicos y profesionales que fueran capaces de administrar y comercializar dichos productos y de

acuerdo al Censo Nacional de 1963, en los inicios del período de industrialización dependiente arrojó la cifra de 991,943 personas con 10 años y más en el país; de esta cifra solamente el 5\% (50,000 personas) había aprobado la primaria y solamente 6,259 habían logrado una educación superior al nivel medio. La demanda de profesionales en el país estaba cubierta en un 19.6\%. La gran pregunta es ¿Por qué tan pocos estudiantes?,¿Cuál era la política del gobierno?

Completado con los graduados en el exterior. La Universidad se convierte entonces en una instancia reproductora de conocimientos y técnicas requeridos por los empresarios de la naciente "industrialización" del país. La gráfica anterior señala que los graduados del periodo, todos a excepto de los ingenieros estaban orientados a brindar servicios, eran empleados de la empresa privada o del estado (Estas empresas necesitaban personal si es posible fueran analfabetas y técnicos medio, como mano de obra barata, en realidad un cortador de algodón y de café no necesitaba preparación).

En la década del 60 al 70 surge una proliferación de carreras y centros de estudios superiores que tratan de dar respuestas a los empresarios que crecían con sus negocios. Entre 1960 y 1969 la Universidad pasa de ofrecer 9 carreras a 20, y en 1978 el número de carreras era de 36.

Desde 1960 hasta 1978 la red de Centros de Educación Superior (CES) llegan a once:

1. La Universidad Autónoma de Nicaragua (UNAN);

2. Ia Universidad Centroamericana, (UCA);

3. Universidad Politécnica (UPOLI);

4. Universidad Privada Autónoma - Centro de Estudios superiores (UPACES),

5. Escuela Nacional de Agricultura y Ganadería (UNAG),

6. Instituto Centroamericano de Administración de Empresas (INCAE),

7. Escuela Nacional de Enfermería (ENE),

8. Instituto Tecnológico de Estudios Superiores (ITESNIC), 
9. Escuela de Contadores Públicos de Nicaragua (ECPN),

10. Centro de Ciencia Comerciales (CCC)

11. y el Centro Nacional de Educación y Ciencias (CENEC).

Como pueden observar la evolución de la universidad ha sido posible gracias a varios factores que se han conjugado, primero la disposición de la Corona en otorgar el beneplácito para instalar la universidad en Nicaragua; contar con los profesionales en el momento histórico que precisamente hicieron su propia historia en función de sacar adelante a la universidad. La independencia de Centroamérica y resto del continente americano, no se hizo esperar y comenzaron a organizar la universidad en facultades, lecciones y las propias carreras con sus planes de estudios.

Ya el Estado de mejor manera y más organizado tomó carta en el asunto para administrar la universidad, que anteriormente estaba a cargo de iglesia. Los cambios fueron sustanciales, sin embargo, se contaba con una universidad centralizada, respondía también a las élites de los gobernantes, las carreras no respondían a las necesidades del estado, de la nación recién conformada.

Pero dialécticamente la universidad deja atrás, lo que no sirve y sale adelante con reformas y desarrollo en áreas organizativas de carácter académicas. Es decir, se cumple con la ley de la negación y así este contexto da paso a nuevos estadios de desarrollo de las universidades en América Latina y el Caribe y específicamente en la Universidad Nicaragüense.

\section{La Reforma de Córdoba en 1918.}

Un salto cualitativo en la vigencia de la universidad, fue la autonomía, un viejo sueño de estudiantes y profesores por muchos años no sólo en Nicaragua sino también en toda América Latina y el Caribe, sobre todo desde el movimiento de Córdoba en 1918. En Nicaragua merecen destacarse los líderes estudiantiles de las jornadas de 1944-47 quienes, ante las violentas intervenciones del ejército en la universidad repetidas veces, urgieron la necesidad de separar de la estructura estatal, la dirección y administración de la universidad. En ese momento el lema era: "Luchemos por la Universidad Central Unida, Autónoma y Popular". Este objetivo estuvo siempre presente en las demandas estudiantiles y fue solicitada también por autoridades universitarias e incluso parlamentarios. Es digno de recordar la gesta histórica estudiantil del 23 de julio del 59, en esa masacre hay 4 muertos y 41 heridos, jóvenes que demandaban con premura la autonomía de la universidad, entre ellos Carlos Fonseca Amador padres del FSLN.

La defensa de la autonomía siguió su lucha hasta el año 2006 con el último presidente neoliberal Ing. Enrique Bolaños antecedidos por el Dr Arnoldo Alemán y la Sra Violeta Barrios de chamorro, gobernaron durante 19 años después del triunfo revolucionario y nunca reconocieron la autonomía universitaria, principalmente lo relativo al $6 \%$ del Presupuesto general de la república que constitucionalmente le corresponde a las universidades públicas; lo único que mantenían era la represión estudiantil, el cerco de las universidades públicas y políticamente desconocimiento del 6\%. Escribieron con sangre esos 19 años, profesores y estudiantes de secundaria, junto la población algunos héroes vivientes, mutilados, ciegos, paralíticos y muchos muertos, es la triste historia del periodo neoliberal gobernado por el Partido Liberal entre 1990 al 2006. Situación que se resuelve con la toma del poder del FSLN y sus aliados en unidad y reconciliación nacional, gobierno que responsablemente ha cumplido con la asignación presupuestaria hasta hoy. 
A partir del año 1990 se aprueba la Ley 89 o ley de autonomía de las instituciones de

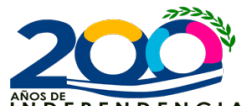
educación superior.

1. Autonomía docente o académica: implica que pueden por si misma nombrar y remover el personal docente y académico, por medio de los procedimientos y requisitos que ellas mismas señalen; seleccionar a sus alumnos, mediante las pruebas y condiciones necesarias; elaborar y aprobar sus planes y programas de estudios y de investigación, etc.

2. Autonomía Orgánica: implica que proceden libremente a integrar sus distintos órganos de gobierno y a elegir sus autoridades.

Con ese espíritu orgánico jurídico y las reivindicaciones provenientes de la Reformas de Córdoba (Argentina) se configura el máximo órgano de gobierno colegiado (Consejo Universitario) y en el que se integran las autoridades superiores Rectores y Vicerrectores, Decanos, Estudiantes y Representantes gremiales... ."parte del Libertad, autonomía y participación constituyeron el eje de las demandas promovidas por los estudiantes durante la reforma universitaria entre 1918 y 1919 en el continente americano. Cien años después, las instituciones de educación superior han incorporado un cuarto propósito: la calidad". Del Huerto. E. (2018).

Figura $N^{\circ}$ 2. Consejo de Gobierno Universitario en Universidades Públicas de Nicaragua.

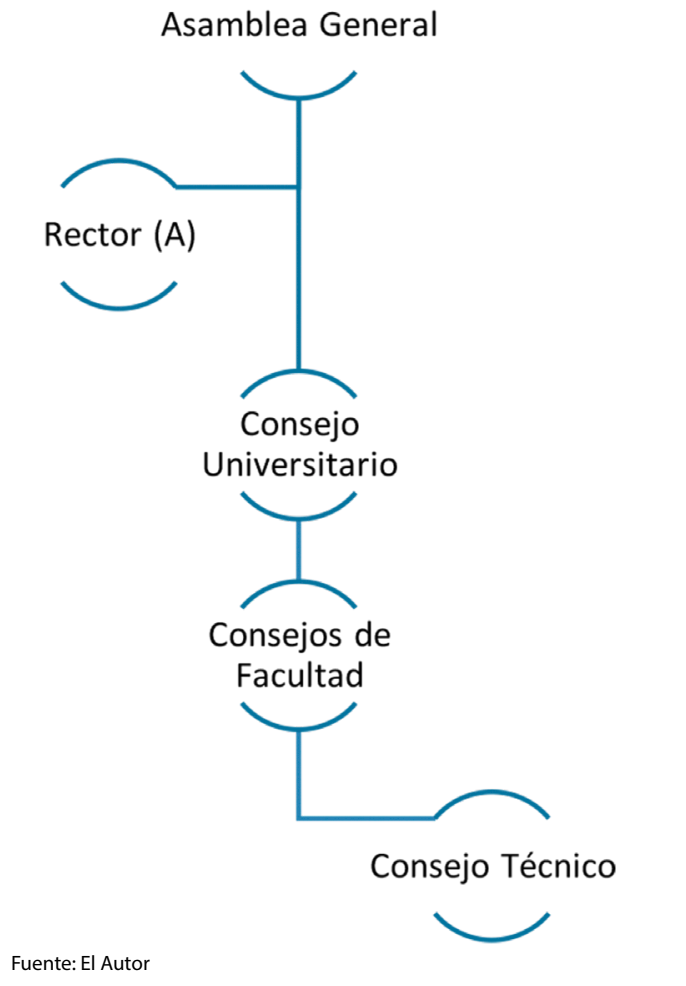

Los órganos de gobierno que las caracterizan hoy son compatibles con lo expresado en el Manifiesto de Córdoba ((Co-gobierno), donde la Asamblea general es la máxima autoridad universitaria sobre el rector, El Consejo Universitario presidido por el Rector (a), los Consejo de Facultades y los consejos departamentales o técnicos. En todas estas instancias de gobierno tienen la misma nomenclatura organizativa, integradas por autoridades universitarias, Decanos, Líderes estudiantiles, líderes gremiales de trabajadores docentes y no docentes.

Así lo corrobora la Ley 89 o de autonomía de las universidades públicas de Nicaragua: Capítulo II. El Consejo Universitario. Artículo 15.- El Consejo Universitario es el máximo órgano de gobierno de la Universidad; estará presidido por el Rector y estará integrado además, por el Vice-Rector General, los decanos de facultad, el Secretario General de la Universidad, que actuará como Secretario del mismo, los Presidentes de las asociaciones estudiantiles de la facultad, el Presidente de la Unión Nacional de Estudiantes de Nicaragua en la Universidad respectiva, dos representantes de la Asociación de Trabajadores Docentes y el Secretario General del Sindicato de Trabajadores no Docente. 
En estas instancias se discuten las demandas que la comunidad educativa plantea a las autoridades superiores; Aspectos académicos en general, administrativos principalmente los relacionados con el uso y rendición de cuentas del presupuesto, problemas gremiales, extensionistas, nacionales o internacionales, pero sobre todo prever, garantizar el futuro próximo de cara a la agenda 2030.

Hoy la mayoría, sino todas las universidades de Centroamérica y A. L y el C han pasado a lo largo de la historia desarrollando un modelo curricular por objetivos, que estuvo vigente por más de 200 años. De igual modo la mayoría están transitando por un Modelo curricular por competencias, vinculantes con las ODS, a la Agenda 2030 y a los Planes de desarrollo de cada país, en Nicaragua al Plan Nacional de Desarrollo Humano.

Nicaragua hoy cuenta con siete universidades públicas: Universidad Nacional Autónoma de Nicaragua, Managua. UNAN-Managua, Universidad Nacional Autónoma de Nicaragua. UNAN-León, Universidad Nacional de Ingeniería (UNI), Universidad Nacional Agraria (UNA), Universidad de las Regiones Autónomas de la Costa Caribe Nicaragüense, URACCAN y la Bluefields Indian \& Caribbean University (BICU)

En Argentina la Universidad de Córdoba fue la primogénita en emanciparse en cuanto a plantear las demandas que por siglos Latinoamérica había manifestado ante los gobiernos caracterizados por mantener bajo su dirección la educación y la educación superior. Una educación a manos del gobierno no para el pueblo, sino para las élites de la iglesia, los latifundistas, los gobernantes y la clase alta. El manifiesto de córdoba se centra en reivindicar en principio la Autonomía, para que sean autoridades propias y elegidos por la misma universidad quienes dirijan los designios de la universidad en materia académica ya administrativa. La forma en que estaba funcionando atentaba contra la funcionalidad de la misma y contra la calidad. Fueron los universitarios, el estudiantado, el profesorado acompañado de la población, personalidades ligadas al que hacer universitario quienes lucharon por décadas hasta obtener la autonomía en 1812.

Respondiendo a la pregunta introductoria inicial, hay que tomar en cuenta que Los tiempos y las instituciones han cambiado y las instituciones. A 103 años del Manifiesto de Córdoba y a 200 de la Independencia de Centroamérica y a 209 del surgimiento de la universidad, la universidad moderna debe estar abierta a la coyuntura política y social, "busca como garantizar que exista una gran cantidad de corrientes de pensamiento y tendencia sin censura, ni prejuicios...."Con un cambio en la filosofía, la conducta y la consciencia está en las manos de los jóvenes forjar la nación mediante la creatividad, la innovación y el emprendimiento que permitan transformar no solo la universidad sino la sociedad.

Algunas instituciones universitarias hoy evidencian una democracia participativa y un profundo sentido social. Participativo puesto que en la mayoría de ellas existe Co-gobierno en el que participan y deciden, los estudiantes, gremios de profesorado y trabajadores y la autoridad universitaria, es una amalgama de tendencias, opiniones, sinergias y trabajo d equipo que se suelen practicar de manera democrática en las distintas formas de gobierno de la universidad. ¿Acaso no era esta misma demanda de la independencia de los pueblos iberoamericanos, Centroamericanos? Vivir en libertad, practicar la democracia, elegir a sus propias autoridades, ¿constituirse por sus propias formas de gobierno para elegir a Gobernadores y diputados y oros funcionarios de gobierno? En este caso fueron casi 500 años bajo la corona española, es decir, bajo discriminación, explotación, racismo, exterminio de la cultura nuestra, saqueo, esta práctica detonaron en el pueblo para vitorear la independencia. 


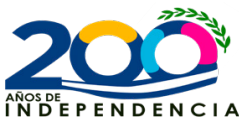

Las estrategias y modelos curriculares emergentes en las universidades de América Latina y el Caribe, persiguen consolidar la filosofía del manifiesto de córdoba y de la independencia de Centroamérica en el sentido de hacer ciudades democráticas, progresistas, independientes, creativas, innovativas, emprendedora, con autonomía en todo el sentido de la palabra y que se ajuste a las demandas de la sociedad, del contexto social, formado profesionales competentes, ciudadanos comprometidos con el desarrollo social.

El Manifiesto de Córdoba considero que abre y mantiene el debate abierto, permanente en los órganos de gobierno, con el estado, con las empresas, con los miembros mismos que la conforman, para buscar entre todos, el desarrollo que todos anhelamos. La universidad latinoamericana moderna, debe apostar por ser una institución abierta, científica, libre, crítica, y en especial con un amplio sentido social.

Cuando en 1937 el primer Somoza, Anastasio Somoza García toma el poder, la matrícula de la Universidad escasamente alcanzaba los 300 alumnos, distribuidos en las carreras que podríamos llamar de prestigios social: Derecho, Medicina, Farmacia. La Universidad y todo el sistema educativo del país funcionaba al margen de las necesidades fundamentales de la población y al margen también del sistema económico caracterizado por una economía agrícola.

Al gobierno de Somoza le interesaba acercarse a la universidad por conveniencia, no por otra cosa. Por un lado, dar la cara de contar con una universidad a nivel de país y por otro lado constar con una instancia como la universidad y la sociedad tenían acceso a ella.(Las élites ligadas al poder eran las que tenían ese acceso)

A partir del 19 de julio de 1979, otros saltos cualitativos y cuantitativos de importancia se dan con el nuevo gobierno Sandinista, convertir la educación en un factor de transformación del país y revertir la situación heredada en el campo educativo era una de las tareas sin duda alguna prioritarias del nuevo gobierno. A simple vista se evidenciaba la necesidad de transformaciones profundas y radicales. El reto de reconstrucción el país y del aporte a la construcción de un hombre nuevo alcanzaba la universidad, pero se carecía de organismos adecuados de planificación para reordenar todo el sistema de Educación Superior.

La proliferación de carreras y centros creados con fines lucrativos o simplemente de prestigio social había sido abusivo y era necesario en ese momento, pensar en una instancia que implemente la redefinición de principios y objetivos que sirviera de base a la reorientación del sistema universitario. El 29 de febrero de 1980 el gobierno de reconstrucción nacional, crea el consejo nacional de educación superior (CNES) como organismo rector de la Educación superior encargado de implementar las transformaciones pertinentes. Este organismo estaba integrado por el Dr. Sergio Ramírez M. Presidente del organismo, (hoy vicepresidente de la República) y los rectores de las universidades (UNAN y UCA para esa fecha).

En el año 1979 había en el país 11 centros de Educación Superior, 10 en Managua y 1 en León, -la Universidad Nacional- con núcleos en Managua, Carazo, Blufields, Puerto Cabezas, que ofrecían carreras similares en cada uno de los centros. Era necesario urgentemente buscar una salida racional a este caos. Reordenar los centros existentes y transformar los currículos de las carreras para un país que necesitaba forman aceleradamente cuadros técnicos y profesionales capaces de poner a andar todas sus instituciones, fue la primera tarea del CNES. Los criterios que se siguieron para la reestructuración fueron evitar duplicidad de 
carreras innecesarias, reagrupar centros buscando racionalizar los escasos recursos del país y estructurar los contenidos e los planes de estudios, de los programas y asignaturas, de acuerdo a la nueva realidad político-social-económica que vivían el país y de acuerdo a la tecnología con la que se podía contar.

En el 80 se creó el centro universitario regional del norte de Estelí, en el 82 la Escuela de Enfermería de la Trinidad (EET-Estelí) y la Escuela de Enfermería de Puerto Cabezas en Zelaya Norte (EEPC-Zelaya).

Un avance significativo ha sido el esfuerzo de implementar una nueva metodología que permitiera la vinculación del estudiante con el campo de trabajo del ramo de su especialidad. El 18 de noviembre de 1982 la Junta de Gobierno decretó la Ley de fomento y Promoción de las Prácticas de Producción en la Educación Superior que en su artículo uno señala: "Se declara de interés social y público el fomento y la promoción de las actividades dirigidas a vincular a los profesores y alumnos de la educación superior con los diferentes sectores de la sociedad y la economía nicaragüense, a fin de combinar el estudio con el trabajo, y lograr que adquieran los frutos profesionales, una formación técnica y revolucionaria estrechamente ligada a la realidad nacional".

El 28 de abril de 1982 se decreta la separación de la Universidad Nacional Autónoma, que estaba ubicada en León en dos núcleos o universidades, uno en León y otro en Managua con independencia en rectoría y demás aspectos administrativos y académicos. En 1983 se crea la Universidad Nacional de Ingeniería "Simón Bolívar", (UNISB). Esta Universidad absorbe todas las carreras de Ingeniería que se impartían en la UNAN y en la UCA.

De 1979 a 1984, la matrícula subió de 26,473 estudiantes a 34,100. La ausencia de una política clara antes del triunfo desembocó en la deformación de la estructura de la matrícula y se refleja en los primeros años después del triunfo. Del $53.3 \%$ de los estudiantes que se matricularon en $1979-80$ el $28.4 \%$ lo hizo en carreras no priorizadas. El grupo de Ciencias Económicas tenía una matrícula similar a los grupos de Ciencias Agropecuarias, Ciencias Médicas y Ciencias de la Educación en conjunto.

La política de ofertar más cupos para los estudiantes recién salidos de secundaria se dio a partir del año 2000, situación que se confirma con el siguiente informe: El rector Maestro Francisco Guzmán Pasos (2010) de la UNAN-Managua, informó que el número de clasificados en el último quinquenio es el siguiente: Año 2006: cupos 5 mil 380, clasificados 4 mil 902, 91.11 por ciento; Año 2007: cupos 5 mil 950, clasificados 5 mil 506, 92.54 por ciento; Año 2008: 7 mil 310 cupos, clasificados 7 mil 175, 98.15 por ciento; Año 2009: 8 mil 072 cupos, clasificados 7 mil 528, 93.82 por ciento; Año 2010: cupos 7 mil 740, clasificados 7 mil 185, 92.68 por ciento. Un total de 34 mil 472 cupos y 32 mil 596 clasificados con un promedio total de 93.68 por ciento.

Según datos del CNU (2018), el total de estudiantes matriculados en las universidades miembros del CNU suman 127,001, de ellos, 69, 903 es decir el 55.04\% son del género femenino. Esto es una muestra de asumir los retos establecidos en la ODS y la AGENDA 2030, en lo que señala con respecto a equidad de género, a la apertura de cupos para todos los estudiantes aspirantes a estudiar en la universidad pública. La Universidad pública del país es gratuita, de calidad y con enfoque humanista. 


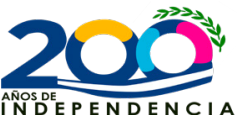

Dentro de la política de expansión educativa se crea en 1980 la Facultad Preparatoria adscrita a la UNAN de Managua y León, con el fin de incorporar a los alumnos de bajos recursos y a jóvenes campesinos de zonas alejadas a niveles superiores de ecuación. En esta facultad se imparte educación preuniversitaria en menor tiempo que el requerido por los planes de estudio del sistema regular de educación. La duración es de 3 años finalizados éstos, el estudiante puede optar por las carreras de Ciencias Médicas, Ciencias de la Educación y Ciencias Agropecuarias que son las carreras priorizadas en todo el subsistema de Educación Superior. Actualmente cursan estudios en la facultad preparatoria 1,388 estudiantes. De ellos el $95 \%$ goza de beca total (alojamiento, alimentación, etc.) y el $5 \%$ tiene becas parciales.

En el año 1980 decenas de estudiantes nicaragüenses salen del país a cursar estudios superiores en universidades de distintos países. Nicaragua está urgida de preparar cuadros técnicos y profesionales en ramas específicas que el país no tiene y que en la difícil situación económica no está en capacidad de implementar.

El programa se va ampliando en la medida en que la solidaridad de gobierno o universidades hermanadas a la Universidad nicaragüense, se va haciendo efectiva. Países como Alemania Federal, Alemania Democrática, Checoslovaquia y otros países de Europa, ofrecieron amplia colaboración. Universidades como la de Barcelona y la Facultad de Ingeniería en España tiene programas y apoyan de diversos modos la Educación Superior en Nicaragua y la mil veces solidaria Cuba puso a disposición del estudiantado nicaragüenses sus centros de estudio. Actualmente son 245 las especialidades con las que cuenta la universidad nicaragüense para enviar a sus estudiantes al exterior. Tecnología, Química de materiales plásticos, Genética animal, Geología, Tecnología del transporte, Perfeccionamiento de terreno, son algunas de ellas. Envío No 57. (1986)

La declaración mundial de la Unesco (1998) sostiene que la calidad de la enseñanza debe "comprender todas las funciones y actividades" de la educación superior del siglo XXI. En respuesta a tal requerimiento han surgido una serie de organismos públicos y privados encargados de realizar los procesos de evaluación y acreditación de esta enseñanza (en nuestro país lo realiza el CNEA. Ley 704).

Ante esta situación, la acreditación se plantea como un medio para lograr el aseguramiento de estándares de calidad en los servicios ofrecidos. Sin embargo, no es el fin del camino. Más bien, debe ser el punto de partida para lograr un objetivo mayor: aquel que persigue la mejora continua.

El Bicentenario patrio es el momento adecuado para repensar sobre el futuro que las instituciones de educación superior, y que asuma los retos que permitan la mejora continua hacia la Calidad de la educación de manera holística, formado profesionales ciudadanos competentes y que resuelvan problemas sociales y laborales bajo la incertidumbre.

Perspectiva de la universidad para el 2030.

En conferencia de prensa, la maestra Ramona Rodríguez Pérez, Rectora de la UNAN-Managua y Presidenta del CNU, en compañía de autoridades de las universidades miembros del Consejo, expuso las perspectivas educativas para el año 2021, entre las que destacó argumenta que la prioridad de las políticas educativas en la región debe ser (Marcados con asterisco propuestas del autor): 
1. Seguir fortaleciendo la calidad de la educación por medio de la formación de profesionales comprometidos con el bienestar del pueblo nicaragüense y de los pueblos latinoamericanos y caribeños.

2. Consolidación de la docencia, la investigación/innovación y la extensión.,

3. Continuar con la ampliación y el aseguramiento de la calidad, del programa Universidad en el Campo (UNICAM)

\begin{tabular}{|c|c|c|c|}
\hline Periodo/Año & $\begin{array}{c}\text { Estructura Organizativa } \\
\text { Universitaria }\end{array}$ & Oferta & Matrícula \\
\hline $\begin{array}{l}1812 \text { a } 1840 \\
\text { Colonia e Independencia de } \\
\text { Centroamérica. }\end{array}$ & $\begin{array}{llr}\text { Delegado } & \text { del } & \text { papa, } \\
\text { máxima } & \text { autoridad } & \text { con } \\
\text { asesores } & \text { académico } y \\
\text { financiero. } & & \end{array}$ & Derecho, medicina y teología & $\begin{array}{l}\text { Matrícula propia de la } \\
\text { élite de los } \\
\text { peninsulares. }\end{array}$ \\
\hline $\begin{array}{l}1847-1878 \quad \text { liberal y } \\
\text { Gobierno Nicaragua } \\
\text { Conservador en Nical }\end{array}$ & $\begin{array}{l}\text { Rector nombrado por la } \\
\text { iglesia }\end{array}$ & $\begin{array}{l}\text { Derecho, medicina, teología y carreras } \\
\text { técnicas }\end{array}$ & $\begin{array}{l}\text { Matrícula contraída } \\
\text { para las élites }\end{array}$ \\
\hline $1960-1995$ & $\begin{array}{l}\text { Rector nombrado por el } \\
\text { estado, a través del } \\
\text { Ministerio de Educación. } \\
\text { Rectorado, con un enfoque } \\
\text { centralista de la } \\
\text { administración educativa, } \\
\text { en los años (1893-1909) } \\
\text { se realizó una reforma } \\
\text { administrativa, pasando al } \\
\text { modelo francés conocido } \\
\text { como Napoleónico }\end{array}$ & $\begin{array}{l}\text { Desde } 1960 \text { hasta } 1978 \text { la red de } \\
\text { Centros de Educación Superior (CES) } \\
\text { llegan a once: } \\
\text { 1. La Universidad Autónoma de } \\
\text { Nicaragua (UNAN); } \\
\text { 2. la Universidad Centroamericana, } \\
\text { (UCA); } \\
\text { 3. Universidad Politécnica (UPOLI); } \\
\text { 4. Universidad Privada Autónoma - } \\
\text { Centro de Estudios superiores } \\
\text { (UPACES), } \\
\text { 5. Escuela Nacional de Agricultura y } \\
\text { Ganadería (UNAG), } \\
\text { 6. Instituto Centroamericano de } \\
\text { Administración de Empresas (INCAE), } \\
\text { 7. Escuela Nacional de Enfermería } \\
\text { (ENE), } \\
\text { 8. Instituto Tecnológico de Estudios } \\
\text { Superiores (ITESNIC), } \\
\text { 9. Escuela de Contadores Públicos } \\
\text { de Nicaragua (ECPN), } \\
\text { 10. Centro de Ciencia Comerciales } \\
\text { (CCC) } \\
\text { y el Centro Nacional de Educación y } \\
\text { Ciencias (CENEC). }\end{array}$ & $\begin{array}{l}34,100 \text { estudiantes } \\
\text { matriculados }\end{array}$ \\
\hline $\begin{array}{l}1995-2010 \\
\text { Gobierno del FSLN, } \\
\text { Gobierno Neoliberal y } \\
\text { Gobierno actual del Frente } \\
\text { Sandinista, Reconciliación } \\
\text { y Unidad Nacional. }\end{array}$ & $\begin{array}{l}\text { Autonomía Universitaria. } \\
\text { Ley } 89 \text { dicta normas de } \\
\text { elección de las } \\
\text { autoridades universitarias. } \\
\text { Reforma de Córdoba } \\
\text { (1918). Órganos de } \\
\text { gobierno colegiados en la } \\
\text { jerarquía académica } \\
\text { (Consejo Universitario, } \\
\text { Consejo de Facultad, } \\
\text { Consejos Técncios) }\end{array}$ & $\begin{array}{l}\text { Entre } 1960 \text { y } 1969 \text { las Universidades } \\
\text { miembros del CNU pasa de ofrecer } 9 \\
\text { carreras a } 20 \text {, y en } 1978 \text { el número de } \\
\text { carreras era de } 36\end{array}$ & $\begin{array}{l}\text { Entre } 1950 \text { y } 1957 \text {, en } \\
\text { promedio se } \\
\text { matricularon } 726 \text { La } \\
\text { población de jóvenes } \\
\text { con edad de estudiar } \\
\text { en la universidad } \\
\text { andaba por un } \\
\text { promedio de } 167,572 \\
\text { jóvenes, que la } \\
\text { demanda satisfecha } \\
\text { era apenas el 0.4\%, } \\
\text { Ramírez. S (1971) }\end{array}$ \\
\hline $\begin{array}{l}\text { 2011-2021 Gobierno actual } \\
\text { del Frente Sandinista de, } \\
\text { Reconciliación y Unidad } \\
\text { Nacional }\end{array}$ & $\begin{array}{l}\text { Asamblea General, } \\
\text { Consejo Universitario, } \\
\text { Rectorado, Consejos de } \\
\text { Facultad, Consejos } \\
\text { técnicos }\end{array}$ & $\begin{array}{l}76 \text { carrearas de Grado, } 105 \text { carreras de } \\
\text { grado en UNAN Managua. } \\
\text { Seis universidades públicas con dos } \\
\text { ubicadas en el Caribe de Nicaragua y } \\
47 \text { Privadas. }\end{array}$ & $\begin{array}{l}43,000 \text { estudiantes. } \\
\text { UNAN Managua y } \\
127,001 \text { Nivel de país. } \\
55 \% \text { Mujeres }\end{array}$ \\
\hline
\end{tabular}




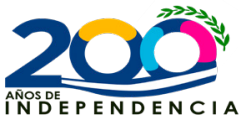

4. Dar continuidad y promover el programa Universidad para la Paz, iniciativa que contribuye a mejorar la convivencia, las relaciones humanas y el diálogo entre la comunidad universitaria.

5. Mantener y ampliar el aseguramiento de los componentes de inclusividad e interculturalidad.

6. Ampliar y promover la movilidad académica y estudiantil nacional e internacional, como estímulo al mejoramiento del rendimiento académico y la eficiencia terminal, además del desarrollo de la interculturalidad como competencia profesional.

7. Apoyar y contribuir con acciones prácticas los programas orientados hacia las políticas públicas, la Educación a Distancia Virtual por medio de la Universidad Abierta en Línea (UALN)

8. Mejorar la infraestructura y el papel de los laboratorios especializados y naturales en la formación de profesionales en diferentes disciplinas.

9. Invertir y ampliar la disposición de la tecnología con la finalidad de generar mejores competencias en los estudiantes y profesores a través de la transformación e implementación de las nuevas tecnologías de la información y la comunicación, la educación continua.

10. Promover que los profesionales dominen y puedan crear programas computacionales

11. Promover e incluir en los planes de estudios el idioma inglés como segunda lengua.

12. Continuar con el avance en la calidad de los procesos educativos con el fin alcanzar la acreditación nacional e internacional de las instituciones y las carreras.

13. La innovación de procesos y productos que dinamicen los emprendimientos universitarios que aportan al Programa Nacional de Nicaragua Creativa.

Finalmente, la Presidenta del CNU comentó sobre la fortaleza del modelo educativo nicaragüense que garantiza la restitución del derecho a la educación en todo el territorio nacional y en todos los niveles, producto de las políticas implementadas por el Estado; "tenemos un gobierno comprometido con las necesidades y aspiraciones de nuestro pueblo, por eso, hoy reafirmamos que desde nuestras universidades continuaremos trabajando por la educación para la vida», expresó la académica.

Fernando Reimers 2015, Citado por L.V. Rivera para lograr la igualdad de oportunidad educativa para estudiantes de distintos grupos sociales. En la actualidad la educación superior en América Central exhibe una trayectoria de limitado apoyo a la movilidad social y al combate de la exclusión social.

Las instituciones de educación superior pueden también ser usadas para apoyar mejorías en todo el sistema educa ív s. Ellas se encuentran cada vez más expuestas a la competencia global y a perder a sus mejores estudiantes y profesores a instituciones extranjeras, especialmente de Estados Unidos. En consecuencia, ellas tienen un verdadero interés en mejorar la calidad, tanto de las instituciones secundarias que las alimentan como de las oportunidades de educación terciaria que proveen.

América Central no necesita circunscribirse al mundo desarrollado en sus planes de reforma educativa. Tampoco debiera planear para hoy, cuando la demanda por educación está cambiando tan dramáticamente. 
Los países ricos tienen sistemas educativos diseñados para desafíos muy diferentes, y eso con frecuencia se nota.

Los fines y objetivos de los sistemas de educación de Centroamérica son muy similares. Todos los países han establecido entre sus objetivos, la formación integral de sus ciudadanos, el cultivo delos valores de solidaridad, respeto a los derechos humanos, respeto a la naturaleza, democracia, convivencia pacífica, respeto a las diversas culturas Lo que diferencia a cada uno de los sistemas educativos es la forma en que deben cumplirse con dichos fines. Eso depende de la concepción ideológica de las fuerzas políticas en el poder y de la conciencia plena de quienes las impulsa y las practica. Nicaragua se apunta por una filosofía humana, solidaria y de práctica cristiana.

\section{Conclusiones}

La Reforma de Córdoba (1918) es el detonante triunfal de la independencia de las Universidades de América Latina y el Caribe, manifiesto que irrumpe con la autonomía administrativa y financiera de las universidades.

Definitivamente la Independencia de Centroamérica (1821) marca el inicio y continuación de la independencia de las universidades en la región y particularmente en Nicaragua.

La inconformidad de los criollos con el trato inhumano de los españoles (En la reconquista) y peninsulares, el desprecio, la discriminación, el atropello a sus derechos sociales laborales, fueron estas las causas que impulsaron al grito de independencia.

La Universidad Nacional es oficializada en el año 1947, 26 años después de la independencia, por el gobierno de Anastasio Somoza García el padre de la dinastía de los Somozas. Es a partir del Rector Pbro Rafael Agustín Ayestas que la universidad da los primeros saltos cualitativos en aspectos académicos, prácticamente Ayestas fue el primer promotor de la movilidad académica al llevar varios estudiantes destacados a Guatemala a formarse.

Luis Somoza Debayle hijo de Anastasio Somoza ya en el poder como presidente de la República, y el Doctor René Schick Gutiérrez su Ministro de Educación Pública fue publicado en La Gaceta N. 73 del 28 de de 1958 la postulación de Universidad Nacional Autónoma de Nicaragua (UNAN) nombrando al Dr Mariano Fiallos Gil padre de la Autonomía Universitaria. (Por conveniencia política ante los ojos de los nicaragüenses, momento de alta tensión social. Fallece en 1964) Le sucedió en la rectoría Carlos Tünnermann Bernheim, hijo del compositor Carlos Tünnermann López y ahijado de Somoza García, le sucedió a este en el cargo Mariano Fiallos Oyanguren en 1974.

El 1982 por decreto de la Junta de Gobierno de Reconciliación Nacional, La Universidad nacional Autónoma de Nicaragua se divide en dos núcleos uno ubicado en León, UNAN León y el otro ubicado en Managua, UNAN Managua.

Las universidades ya han alcanzado un alto grado organizativo, conformada por los distintos órganos de gobierno, integrado por Vicerrectores con funcionas bien delimitadas, se definen las Facultades que atienden en la actualidad áreas profesionales afines y de estas dependen los departamentos docentes con 


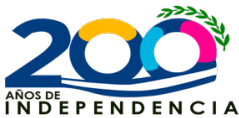

su respectivo órgano de gobierno, el consejo técnico.

La demanda y la oferta hasta el 2020 no respondían parcialmente a las demandas del desarrollo técnicocientífico del país. Sin embargo, a esa misma fecha la matrícula estudiantil en la universidades miembros y adscritas al CNU ronda los 130,000, de ellos 43, 000 es decir el 33\% de la matrícula está en la UNAN Managua, de ese total el $61 \%$ son del género femenino.

La oferta académica en el caso particular de la UNAN Managua, a nivel de pregrado son 4 carreras técnicas propias, aunque otras a nivel de licenciatura dan salida a técnicos superiores. El total de Licenciaturas o profesiones equivalentes ofertadas suman 76 y las carreras ofertadas en el posgrado suman 105.

El desarrollo administrativo y académico es significativo e importante por las universidades públicas, hoy se cuenta con una buena inversión en infraestructura informática, laboratorios, laboratorios de investigaciones de referencias a nivel regional, (CIRA, CIGEO, BIOTECNOLOGÍA), sedes en las regiones bien equipadas, prácticamente cubriendo todo el país.(UNAN Managua y UNAN León), académicamente la formación ha sido un factor de prioridad y desde luego impulsando la calidad de la docencia y la investigación.

Las relaciones externas a nivel nacional e internacional, es una prioridad para contribuir con el cumplimiento de la agenda 2030 y el Plan Nacional de desarrollo Humano, desde luego con el acompañamiento de los estudiantes que finalizan sus carreras y docentes experimentados en la proyección social. Las universidades en Nicaragua y particularmente la UNAN Managua está ejecutando a partir del año 2021 un modelo curricular por competencias y ha entrado al proceso de acreditación a nivel nacional con el CNEA y a nivel internacional con el Consejo de Evaluación y Acreditación Internacional (CEAl) instancia de la UDUAL.

En cuanto a calidad se refiere las universidades deben registrar y analizar con fines de mejora continua los indicadores del Rendimiento académico y eficiencia terminal, tal como lo propone en parte las CRESS (2008)

Creemos en un solo Dios, Padre Todopoderoso, creador de Cielo y Tierra, de todo lo visible e invisible. Creemos en un solo Señor, Jesucristo, Hijo único de Dios, nacido del Padre antes de todos los siglos: Dios de Dios, Luz de Luz, Dios verdadero de Dios verdadero, engendrado no creado, de la misma naturaleza del Padre, por quien todo fue hecho. Que por nosotros y por nuestra salvación bajó del cielo: por obra del Espíritu Santo se encarnó de María, la Virgen y se hizo hombre. Por nuestra causa fue crucificado en tiempos de Poncio Pilato: padeció y fue sepultado. Resucitó al

Desde toda la eternidad, Dios Padre engendra a su Hijo, que es Dios como él y de su misma 


\section{Referencias bibliográficas.}

- $\quad$ Asamblea Nacional de la República de nicaragua. (1980). Ley de Autonomía de la educación superior en Nicragua. En A. N. nicaragua, Ley de Autonomía de la educación superior en Nicragua (pág. 22). Managua: La Gaceta.

- $\quad$ Bernheim, C. T. (26 de 07 de 2021). https://www.enriquebolanos.org/libro/La-Universidad-antelos-retos-del-siglo-XX. Obtenido de https://www.enriquebolanos.org/libro/La-Universidad-ante-los-retosdel-siglo-XX.

- $\quad$ Envío. (1986). La Universidad en Nicaragua: una transformación enmarcha. Envío, 565.

- Lilina Vanessa Rivera Pérez, e. t. (26 de 07 de 2021). http://www.estudioscomparativossistemaeducativo.C.A.com. Obtenido de http://www.estudioscomparativos-sistemaeducativo.C.A.com.

- Marimón, M. E. (26 de 07 de 2021). http://scielo.sld.cu/scielo.php?script=sci_arttext\&pid=S025743142018000100003\&lng=es\&tlng=es. Obtenido de http://scielo.sld.cu/scielo.php?script=sci_ arttext\&pid=S0257-43142018000100003\&lng=es\&tIng=es.

- Medrano, S. H. (26 de 07 de 2021). https://www.buenastareas.com/. Obtenido de https://www. buenastareas.com/.

- Pasos, F. G. (29 de 07 de 2021). https://www.universia.net/es/actualidad/orientacion-academica/ unan-managua-presenta-resultados-ingreso-2010-198896.html. Obtenido de https:/www.universia.net/ es/actualidad/orientacion-academica/unan-managua-presenta-resultados-ingreso-2010-198896.html.

- Pérez, R. R. (27 de 07 de 2021). http://www.cnu.edu.ni/consejo-nacional-de-universidadescomparte-metas-de-la-educacion-superior-para-el-ano-2021/. Obtenido de http://www.cnu.edu.ni/ consejo-nacional-de-universidades-comparte-metas-de-la-educacion-superior-para-el-ano-2021/.

- Ruz, F. C. (26 de 07 de 2021). https://www.radiogritodebaire.cu/politica/fidel-castro-conceptorevolucion/. Obtenido de https://www.radiogritodebaire.cu/politica/fidel-castro-concepto-revolucion/.

- Wikipedia.org. (26 de 07 de 2021). https://es.wikipedia.org/w/index.php?title=Independencia_ de_Centroam\%C3\%A9rica\&oldid=137099675. Obtenido de https://es.wikipedia.org/w/index. php?title=Independencia_de_Centroam\%C3\%A9rica\&oldid=137099675. 